\title{
ON THE ORDER OF ARC-STABILISERS IN ARC-TRANSITIVE GRAPHS, II
}

\author{
GABRIEL VERRET \\ (Received 30 April 2012; accepted 11 May 2012; first published online 2 August 2012)

\begin{abstract}
Let $\Gamma$ be a $G$-vertex-transitive graph and let $(u, v)$ be an arc of $\Gamma$. It is known that if the local action $G_{v}^{\Gamma(v)}$ (the permutation group induced by $G_{v}$ on $\Gamma(v)$ ) is permutation isomorphic to the dihedral group of degree four, then either $\left|G_{u v}\right|$ is 'small' with respect to the order of $\Gamma$ or $\Gamma$ is one of a family of well-understood graphs. In this paper, we generalise this result to a wider class of local actions.
\end{abstract}

2010 Mathematics subject classification: primary 20B25; secondary 05E18.

Keywords and phrases: arc-transitive graphs, graph-restrictive group, local action.

\section{Introduction}

All graphs considered in this paper are finite and simple. A graph $\Gamma$ is said to be $G$ vertex-transitive if $G$ is a subgroup of $\operatorname{Aut}(\Gamma)$ acting transitively on the vertex set $\mathrm{V}(\Gamma)$ of $\Gamma$. Similarly, $\Gamma$ is said to be $G$-arc-transitive if $G$ acts transitively on the arc set $\mathrm{A}(\Gamma)$ of $\Gamma$. (An arc is an ordered pair of adjacent vertices.) When $G=\operatorname{Aut}(\Gamma)$, the prefix $G$ in the above notation is sometimes omitted.

A very early and important result concerning arc-transitive graphs is the beautiful theorem of Tutte [5, 6] that, in a connected 3-valent arc-transitive graph, the order of an arc-stabiliser divides 16 . To consider generalisations of this result, a very fruitful concept is that of local action, which we now define.

Definition 1.1. Let $L$ be a permutation group, let $\Gamma$ be a connected $G$-vertex-transitive graph and let $G_{v}^{\Gamma(v)}$ denote the permutation group induced by the action of $G_{v}$ on the neighbourhood $\Gamma(v)$ of a vertex $v$. Then $(\Gamma, G)$ is said to be locally-L if $G_{v}^{\Gamma(v)}$ is permutation isomorphic to $L$. More generally, if $\mathcal{P}$ is a permutation group property, then $(\Gamma, G)$ will be called locally-P provided that $G_{v}^{\Gamma(v)}$ possesses the property $\mathcal{P}$.

Note that, if $\Gamma$ has valency $d$, then the permutation group $G_{v}^{\Gamma(v)}$ has degree $d$ and, up to permutation isomorphism, does not depend on the choice of $v$. In [7], the author

(c) 2012 Australian Mathematical Publishing Association Inc. 0004-9727/2012 \$16.00 
introduced the following notion: a transitive permutation group $L$ is called graphrestrictive if there exists a constant $c(L)$ such that, for every locally- $L$ pair $(\Gamma, G)$ and an $\operatorname{arc}(u, v)$ of $\Gamma$, the inequality $\left|G_{u v}\right| \leq c(L)$ holds.

This definition makes it possible to give succinct formulations of many results and questions. For example, Tutte's theorem can be restated as follows: the symmetric group $\mathrm{S}_{3}$ in its natural action on three points is graph-restrictive and the constant $c\left(\mathrm{~S}_{3}\right)$ can be chosen to be 16 . The problem of determining which transitive permutation groups are graph-restrictive was also raised in [7]. A survey of the state of this problem can be found in [2].

If $L$ is a transitive permutation group that is not graph-restrictive then, by definition, there exist locally- $L$ pairs $(\Gamma, G)$ with $\left|G_{u v}\right|$ arbitrarily large. In this case, the best that we can hope for is to obtain an upper bound on the order of $G_{u v}$ in terms of the order of $\Gamma$.

The smallest transitive permutation group (either by degree or by order) which is not graph-restrictive is $\mathrm{D}_{4}$, the dihedral group of degree four. The locally- $\mathrm{D}_{4}$ case was thoroughly investigated in [3], where it was shown that there exists a sublinear function $f$ such that, if $(\Gamma, G)$ is a locally- $\mathrm{D}_{4}$ pair, then $\left|G_{u v}\right| \leq f(|\mathrm{~V}(\Gamma)|)$, unless $\Gamma$ is one of a family of well-understood graphs. Our goal in this paper is to generalise these results to a wider class of local actions, which we now define.

Definition 1.2. Let $p$ be a prime. A transitive permutation group $L$ on $\Omega$ is weakly $p$ subregular if there exist $x, y \in \Omega$ such that $\left|L_{x}\right|=p$ and $x^{\bar{L}} \cup y^{\bar{L}}=\Omega$, where $\bar{L}=\left\langle L_{x}, L_{y}\right\rangle$ and $x^{\bar{L}}$ (respectively, $y^{\bar{L}}$ ) denotes the orbit of $x$ (respectively, $y$ ) under $\bar{L}$.

This definition generalises the notion of a $p$-subregular permutation group, which is the case when $\bar{L}=L$ (see [7, Definition 1.1]). Weakly $p$-subregular permutation groups are graph-restrictive if and only if they are $p$-subregular. (This follows from [2, Theorem 4 and Corollary 11].)

Some easy examples of weakly $p$-subregular permutation groups which are not graph-restrictive are dihedral groups of even degree and $\mathbb{Z}_{p}$ wr $\mathbb{Z}_{2}$ in its natural action on $\mathbb{Z}_{p} \times \mathbb{Z}_{2}$. There are also some nonsoluble examples, such as $S_{5}$ acting on its 5-cycles by conjugation. Our main goal in this paper is to prove the following theorem.

Theorem A. Let $(\Gamma, G)$ be a locally-L pair and let $(u, v)$ be an arc of $\Gamma$. If $L$ is weakly p-subregular for some prime $p$ then either:

(1) $\left|G_{u v}\right| \leq|\mathrm{A}(\Gamma)|^{3} ;$ or

(2) L is permutation isomorphic to $\mathbb{Z}_{p} \mathrm{wr} \mathbb{Z}_{2}$ and $\Gamma \cong \mathrm{C}(p, r, s)$ for some $r \geq 3$ and $1 \leq s \leq r-1$.

We now define the graphs $\mathrm{C}(p, r, s)$ which appear in Theorem A. These were first studied by Praeger and Xu [4]. Let $p$ be a prime and let $r$ and $s$ be positive integers with $r \geq 3$ and $1 \leq s \leq r-1$. Let $\mathrm{C}(p, r, 1)$ be the lexicographic product $\mathrm{C}_{r}\left[p K_{1}\right]$ of a cycle of length $r$ and an edgeless graph on $p$ vertices. In other words, $\mathrm{V}(\mathrm{C}(p, r, 1))=\mathbb{Z}_{r} \times \mathbb{Z}_{p}$ with $(i, u)$ being adjacent to $(j, v)$ if and only if $i-j \in\{-1,1\}$. A path in $\mathrm{C}(p, r, 1)$ is 
called traversing if it contains at most one vertex from $\{y\} \times \mathbb{Z}_{p}$ for each $y \in \mathbb{Z}_{r}$. For $s \geq 2$, let $\mathrm{C}(p, r, s)$ be the graph with vertices being the traversing paths in $\mathrm{C}(p, r, 1)$ of length $s-1$ and with two such $(s-1)$-paths being adjacent in $\mathrm{C}(p, r, s)$ if and only if their union is a traversing path in $\mathrm{C}(p, r, 1)$ of length $s$. Clearly, $\mathrm{C}(p, r, s)$ is a connected $2 p$-valent graph with $r p^{s}$ vertices.

There is an obvious action of the wreath product $H=\mathbb{Z}_{p} \mathrm{wr}_{r}$ as a group of automorphisms of $\mathrm{C}(p, r, 1)$ which induces a faithful arc-transitive action on $\mathrm{C}(p, r, s)$ for $s \leq r-1$. It is easily seen that, in this case, the local action is $\mathbb{Z}_{p}$ wr $\mathbb{Z}_{2}$ in its natural action on $\mathbb{Z}_{p} \times \mathbb{Z}_{2}$, which is weakly $p$-subregular. Note that $|H|=2 r p^{r}$ and hence the order of the stabiliser of an $\operatorname{arc}$ of $\mathrm{C}(p, r, s)$ in $H$ is $p^{r-s-1}$. Thus, if $p$ and $s$ are fixed, then the order of the stabiliser in $H$ of an arc of $\mathrm{C}(p, r, s)$ grows exponentially with $r$ and hence exponentially with the number of arcs of $\mathrm{C}(p, r, s)$. This behaviour contrasts sharply with the polynomial upper bound which is the other possibility in the conclusion of Theorem A.

\section{Proof of Theorem A}

In the course of the proof, we will need the following result of Glauberman.

Theorem 2.1 [1, Theorem 1]. Let $P$ be a subgroup of a finite group $G$, let $g \in G$, and let $P \cap P^{g}$ be a normal subgroup of prime index $p$ in $P^{g}$. Let $n$ be a positive integer, and let $\tilde{G}=\left\langle P, P^{g}, \ldots, P^{g^{n}}\right\rangle$. Assume that:

(1) $g$ normalises no nonidentity normal subgroup of $P$; and

(2) $P \cap Z(\tilde{G})=1$.

Then $|P|=p^{t}$ for some positive integer $t$ for which $t \leq 3 n$ and $t \neq 3 n-1$. Moreover, if $n=2, p=2$ and $t=6$, then $P$ contains a nonidentity normal subgroup of $\tilde{G}$.

We now note a few elementary facts about weakly $p$-subregular permutation groups. Let $L$ be a permutation group on $\Omega$. Let $p$ be a prime and suppose that $L$ is weakly $p$-subregular as witnessed by $x$ and $y$. Let $\bar{L}=\left\langle L_{x}, L_{y}\right\rangle$. As $x^{\bar{L}} \cup y^{\bar{L}}=\Omega$, the group $\bar{L}$ contains all point-stabilisers of $L$ and hence $\bar{L}$ is normal in $L$. Since $L_{x}$ and $L_{y}$ both have order $p, L_{x y}$ is normal in both of them and hence $L_{x y}$ is normal in $\bar{L}$. Since $\Omega=x^{\bar{L}} \cup y^{\bar{L}}$, it follows that $L_{x y}=1$ and $L_{x} \neq L_{y}$. We are now ready to prove the following theorem about locally weakly $p$-subregular pairs.

Theorem 2.2. Let $p$ be a prime, let $(\Gamma, G)$ be a locally weakly p-subregular pair and let $(u, v)$ be an arc of $\Gamma$. Then either $\left|G_{u v}\right| \leq|\mathrm{A}(\Gamma)|^{3}$ or $G$ contains an abelian normal subgroup intersecting $G_{u v}$ nontrivially.

Proof. Fix a vertex $v$ of $\Gamma$. By definition, there exist $u, w \in \Gamma(v)$ such that $\left|G_{u v}^{\Gamma(v)}\right|=p$ and $\Gamma(v)=u^{\bar{X}} \cup w^{\bar{X}}$, where $\bar{X}=\left\langle G_{u v}^{\Gamma(v)}, G_{v w}^{\Gamma(v)}\right\rangle$. By the notes preceding this theorem, $\bar{X}$ is normal in $G_{v}^{\Gamma(v)}, G_{u v w}^{\Gamma(v)}$ is normal and of index $p$ in both $G_{u v}^{\Gamma(v)}$ and $G_{v w}^{\Gamma(v)}$, and $G_{u v}^{\Gamma(v)} \neq G_{v w}^{\Gamma(v)}$.

The fact that $L$ is transitive implies that $\Gamma$ is $G$-arc-transitive and hence there exists $g \in G$ such that $(u, v)^{g}=(v, w)$. Let $P=G_{u v}$ and $X=\left\langle P, P^{g}\right\rangle=\left\langle G_{u v}, G_{v w}\right\rangle$. Both $P$ and 
$P^{g}$ contain the kernel of the action of $G_{v}$ on $\Gamma_{v}$. It follows from the previous paragraph that $X$ is normal in $G_{v}$, that $\Gamma(v)=u^{X} \cup w^{X}$, that $P \cap P^{g}$ is normal and of index $p$ in both $P$ and $P^{g}$, and that $P \neq P^{g}$.

For any vertex $\alpha \in \mathrm{V}(\Gamma)$, there exists $f \in G$ such that $v^{f}=\alpha$. Let $X[\alpha]=X^{f}$. Note that since $X$ is normal in $G_{v}, X[\alpha]$ only depends on $\alpha$ and not on the choice of $f$. Moreover, $X[\alpha]$ is a normal subgroup of $G_{\alpha}$. Finally, let $G^{*}=\langle g, P\rangle$. We prove the theorem by a sequence of claims.

Claim 1. $G^{*}$ is vertex-transitive.

First, note that $G^{*}=\langle g, X\rangle$. For $i \in \mathbb{Z}$, let $v_{i}=v^{g^{i}}$ and let $X_{i}=X^{g^{i}}=X\left[v_{i}\right]$. Note that $\left(v_{-1}, v_{0}, v_{1}\right)=(u, v, w)$ and hence $\Gamma\left(v_{i}\right)=v_{i-1}^{X_{i}} \cup v_{i+1}^{X_{i}}$ for any $i \in \mathbb{Z}$. Let $X^{*}=\left\langle X_{i} \mid i \in \mathbb{Z}\right\rangle$ and let $S=v^{\langle g\rangle}=\left\{v_{i} \mid i \in \mathbb{Z}\right\}$. Note that $X^{*} \leq G^{*}$, hence it suffices to show that $S^{X^{*}}=$ $\mathrm{V}(\Gamma)$.

Suppose, for a contradiction, that there exists a vertex not in $S^{X^{*}}$ and choose one with minimum distance to $S$. Call this vertex $\alpha$ and let $\left(p_{0}=\alpha, \ldots, p_{n-1}, p_{n}=v_{i}\right)$ be a shortest path from $\alpha$ to a vertex of $S$. Since $\Gamma\left(v_{i}\right)=v_{i-1}^{X_{i}} \cup v_{i+1}^{X_{i}}$, it follows that there exists $\sigma \in X_{i} \leq X^{*}$ such that $p_{n-1}^{\sigma} \in\left\{v_{i-1}, v_{i+1}\right\} \subseteq S$. Since $\alpha$ is not in $S^{X^{*}}$, neither is $\alpha^{\sigma}$, but $\alpha^{\sigma}$ is closer to $S$ than $\alpha$ is, which is a contradiction.

Claim 2. $g$ normalises no nonidentity normal subgroup of $P$.

Any normal subgroup $N$ of $P$ that is normalised by $g$ must be normalised by $\langle g, P\rangle=G^{*}$ which is vertex-transitive. Since $N$ fixes $v$, it must fix every vertex and hence be trivial.

For $i \geq 0$, let $H_{i}=\left\langle P, P^{g}, \ldots, P^{g^{i}}\right\rangle$. Let $n$ be the smallest integer such that $H_{n}=$ $H_{n+1}$.

Claim 3. $H_{n}$ is normal in $G$.

First, note that $\left(H_{n}\right)^{g} \leq H_{n+1}=H_{n}$ and hence $g$ normalises $H_{n}$. Recall that $P \neq P^{g}$, which implies that $n \geq 1$ and $H_{n}=\left\langle X, X^{g}, \ldots, X^{g^{n-1}}\right\rangle$. Since $H_{n}$ is normalised by $\langle g, P\rangle=G^{*}$ which is vertex-transitive, it follows that $H_{n}=\langle X[\alpha] \mid \alpha \in \mathrm{V}(\Gamma)\rangle$. Since $X=X[v]$ is normal in $G_{v}$, it follows that $\langle X[\alpha] \mid \alpha \in \mathrm{V}(\Gamma)\rangle$ is normal in $G$.

Let $N$ be the normal closure of $P \cap \mathrm{Z}\left(H_{n}\right)$ in $G$. Since $H_{n}$ is normal in $G$, so is $\mathrm{Z}\left(H_{n}\right)$. It follows that $N \leq \mathrm{Z}\left(H_{n}\right)$ and hence $N$ is abelian. If $N \neq 1$, then $N \cap P \neq 1$ and $N$ is the required normal subgroup of $G$ intersecting $G_{u v}$ nontrivially.

We may thus assume that $N=1$ and hence $P \cap \mathrm{Z}\left(H_{n}\right)=1$. As we noted earlier, $P \cap P^{g}$ is normal of index $p$ in both $P$ and $P^{g}$. We can use Theorem 2.1 with $\tilde{G}=H_{n}$ to conclude that $|P|=p^{t}$ for some $t \leq 3 n$. In particular, $P$ is a $p$-group.

Claim 4. $\left|H_{n}\right| \geq p^{n}|P|$.

Let $i \in\{1, \ldots, n\}$. If $P^{g^{i}}$ is a subgroup of $H_{i-1}$, then $H_{i-1}=H_{i}$, contradicting the minimality of $n$. It follows that $P^{g^{i}}$ is a subgroup of $H_{i}$ but not of $H_{i-1}$. Since $P$ is a $p$-group, so is $P^{g^{i}}$ and hence $\left|H_{i}\right| \geq p\left|H_{i-1}\right|$. The claim then follows by induction. 
Since $\left|G_{u v}\right||\mathrm{A}(\Gamma)|=|G| \geq\left|H_{n}\right| \geq p^{n}|P|$, it follows that $|\mathrm{A}(\Gamma)| \geq p^{n} \geq p^{t / 3}$, concluding the proof.

One of the reasons why Theorem 2.2 is interesting is that the condition of admitting an abelian normal subgroup which intersects an arc-stabiliser nontrivially is surprisingly strong. In fact, as we will see in the rest of this section, in the weakly $p$-subregular case it is enough to completely determine the structure of $G_{v}^{\Gamma(v)}$ (see Lemma 2.4) and almost completely determine the graph! We first show some of the 'local' consequences of this condition in general.

Lemma 2.3. Let $L$ be a transitive permutation group, let $(\Gamma, G)$ be a locally-L pair, let $(u, v)$ be an arc of $\Gamma$ and suppose that $G$ contains an abelian normal subgroup intersecting $G_{u v}$ nontrivially. Then, L contains an abelian normal subgroup which is not semiregular.

Proof. Suppose, for a contradiction, that each abelian normal subgroup of $L$ is semiregular and let $N$ be an abelian normal subgroup of $G$ with $N_{u v} \neq 1$. Since $N$ is an abelian normal subgroup of $G, N_{v}$ is an abelian normal subgroup of $G_{v}$ and hence $N_{v}^{\Gamma(v)}$ is an abelian normal subgroup of $G_{v}^{\Gamma(v)} \cong L$. It follows that $N_{v}^{\Gamma(v)}$ is semiregular and hence $N_{u v}$ fixes $\Gamma(v)$. It follows that fixing an arc in $N$ fixes all adjacent arcs and, by connectedness, that $N_{u v}=1$, which is a contradiction.

In view of Theorem 2.2 and Lemma 2.3, it is natural to study weakly $p$-subregular permutation groups which admit an abelian normal subgroup which is not semiregular.

LEMMA 2.4. Let $p$ be a prime and let L be a weakly p-subregular permutation group. If $L$ contains an abelian normal subgroup which is not semiregular, then $L$ is permutation isomorphic to $\mathbb{Z}_{p}$ wr $\mathbb{Z}_{2}$.

Proof. Let $\Omega$ be the set on which $L$ is a permutation group. By definition, there exist $x, y \in \Omega$ such that $\left|L_{x}\right|=p$ and $x^{\bar{L}} \cup y^{\bar{L}}=\Omega$, where $\bar{L}=\left\langle L_{x}, L_{y}\right\rangle$. Let $N$ be an abelian normal subgroup of $L$ which is not semiregular. Since $N$ is normal and not semiregular, $N_{x}$ and $N_{y}$ must be nontrivial. Since $\left|L_{x}\right|=\left|L_{y}\right|=p$ is prime, it follows that $N_{x}=L_{x}$ and $N_{y}=L_{y}$ and therefore $\bar{L} \leq N$. Finally, since $N$ is abelian and not semiregular, it is not transitive and hence $\bar{L}=N$.

Since $N$ is abelian, and $L_{x} \neq L_{y}$, it follows that $N=L_{x} \times L_{y} \cong \mathbb{Z}_{p}^{2}$. Since $L$ is transitive, there exists $h \in L$ such that $x^{h}=y$. Write $L_{x}=\langle a\rangle$ and $L_{y}=\langle b\rangle$. Since $\left(L_{x}\right)^{h}=L_{y}$, we have that $a^{h}=b$ and, since $N$ has index 2 in $L$, it follows that $h^{2}$ is contained in $N$ which is abelian and hence $y^{h}=x$ and $b^{h}=a$ and, in particular, $h$ does not commute with either $a$ or $b$.

We now show that there exists an involution in $L \backslash N$. If $h^{2}=1$, then we are done. Otherwise, $h^{2}$ has order $p$. If $p$ is odd, then $h^{p}$ is an involution in $L \backslash N$. If $p=2$, then $h^{2}=a b$ and it is easy to check that $h a$ is an involution in $L \backslash N$.

Let $h^{\prime}$ be an involution in $L \backslash N$. Clearly, $a^{h^{\prime}}=a^{h}=b$ and $b^{h^{\prime}}=a$. This shows that, as an abstract group, $L$ is isomorphic to $\mathbb{Z}_{p}$ wr $\mathbb{Z}_{2}$. Finally, note that $\Omega=x^{N} \cup y^{N}=$ $x^{L_{y}} \cup y^{L_{x}}$, while $h^{\prime}$ interchanges $x^{L_{y}}$ and $y^{L_{x}}$, which concludes the proof. 
Lemma 2.4 will allow us to use the following theorem of Praeger and $\mathrm{Xu}$.

Theorem 2.5 [4, Theorem 1]. Let $p$ be a prime and let $\Gamma$ be a 2 p-valent $G$ arc-transitive graph such that $G$ has an abelian normal p-subgroup which is not semiregular on the vertices of $\Gamma$. Then $\Gamma \cong \mathrm{C}(p, r, s)$ for some $r \geq 3$ and $1 \leq s \leq r-1$.

We are now ready to prove Theorem A, which we restate for convenience.

Theorem A. Let $(\Gamma, G)$ be a locally-L pair and let $(u, v)$ be an arc of $\Gamma$. If $L$ is weakly p-subregular for some prime $p$ then either:

(1) $\left|G_{u v}\right| \leq|\mathrm{A}(\Gamma)|^{3}$; or

(2) L is permutation isomorphic to $\mathbb{Z}_{p} \mathrm{wr} \mathbb{Z}_{2}$ and $\Gamma \cong \mathrm{C}(p, r, s)$ for some $r \geq 3$ and $1 \leq s \leq r-1$.

Proof. By Theorem 2.2, we may assume that $G$ contains an abelian normal subgroup $N$ intersecting $G_{u v}$ nontrivially. By Lemma 2.3, this implies that $L$ contains an abelian normal subgroup which is not semiregular. By Lemma 2.4, it follows that $L$ is permutation isomorphic to $\mathbb{Z}_{p}$ wr $\mathbb{Z}_{2}$. In particular, $L$ has degree $2 p$ and hence $\Gamma$ is $2 p$-valent.

Since $L$ is weakly $p$-subregular, we have that $\left|G_{u v}^{\Gamma(v)}\right|=p$ and hence $G_{u v}$ is a $p$-group. Let $M$ be the group generated by the elements of order $p$ in $N$. This is an elementary abelian $p$-group which is characteristic in $N$ and hence normal in $G$. Since $G_{u v}$ is a $p$ group, it follows that there is an element of order $p$ in $N_{u v}$ and hence $M_{u v} \neq 1$. Finally, by Theorem 2.5, it follows that $\Gamma \cong \mathrm{C}(p, r, s)$ for some $r \geq 3$ and $1 \leq s \leq r-1$.

\section{Further work}

The two main tools in the proof of Theorem A are Theorems 2.2 and 2.5 [4, Theorem 1]. It seems quite likely that the conclusion of Theorem 2.2 holds under a much weaker hypothesis, especially if we are willing to replace the constant 3 in the exponent by a different constant. For example, we make the following conjecture, which would generalise Theorem 2.2.

Conjecture 3.1. For any $k$, there exists a constant $d_{k}$ such that, if $\Gamma$ is $k$-valent and $G$-arc-transitive, and the arc-stabiliser $G_{u v}$ is soluble, then either $\left|G_{u v}\right| \leq|\mathrm{A}(\Gamma)|^{d_{k}}$ or $G$ contains an elementary abelian normal $p$-subgroup intersecting $G_{u v}$ nontrivially.

Note that the solubility of $G_{u v}$ can be checked locally. Indeed, it is not hard to see that $G_{u v}$ is soluble if and only if $G_{u v}^{\Gamma(v)}$ is. A missing piece of the puzzle seems to be our lack of a generalisation of Theorem 2.5. We thus pose the following question.

Question 3.2. What can be said about a $G$-arc-transitive graph $\Gamma$ such that $G$ has an abelian normal $p$-subgroup which is not semiregular on the arcs of $\Gamma$ ?

\section{Acknowledgements}

Most of this work was part of the author's PhD thesis written under the supervision of Primož Potočnik at the University of Ljubljana. We would also like to thank Pablo Spiga for valuable advice. 


\section{References}

[1] G. Glauberman, 'Normalizers of $p$-subgroups in finite groups', Pacific J. Math. 29 (1969), 137-144.

[2] P. Potočnik, P. Spiga and G. Verret, 'On graph-restrictive permutation groups', J. Combin. Theory Ser. B 102 (2012), 820-831.

[3] P. Potočnik, P. Spiga and G. Verret, 'Bounding the order of the vertex-stabiliser in 3-valent vertextransitive and 4-valent arc-transitive graphs', arXiv:1010.2546v1 [math.CO].

[4] C. E. Praeger and M. Y. Xu, 'A characterization of a class of symmetric graphs of twice prime valency', European J. Combin. 10 (1989), 91-102.

[5] W. T. Tutte, 'A family of cubical graphs', Proc. Cambridge Philos. Soc. 43 (1947), 459-474.

[6] W. T. Tutte, 'On the symmetry of cubic graphs', Canad. J. Math. 11 (1959), 621-624.

[7] G. Verret, 'On the order of arc-stabilizers in arc-transitive graphs', Bull. Aust. Math. Soc. 80 (2009), 498-505.

GABRIEL VERRET, FAMNIT, University of Primorska,

Glagoljaška 8, 6000 Koper, Slovenia

e-mail: gabriel.verret@pint.upr.si 